\title{
Frequência de alterações posturais em hemofílicos
}

\author{
Frequency of postural alterations in haemophiliacs
}

\begin{abstract}
Rafaele Serra dos Santos', Giovana Rossi Figueirôa ${ }^{2}$, Brenda de Araujo Machado ${ }^{3}$, Carlos André Gomes Silva Mamede ${ }^{4}$, Lúcia Maria Leandro Gois ${ }^{5}$, Mário Cezar Macedo Silva Junior
\end{abstract}

\author{
${ }^{1}$ Autora para correspondência. Universidade do Estado da Bahia. Salvador, Bahia, Brasil. \\ ORCID: 0000-0001-5647-1248. rafaelleserra@hotmail.com \\ ${ }^{2}$ Universidade do Estado da Bahia. Salvador, Bahia, Brasil. ORCID: 0000-0002-1726-4980. giovana@figueiroa.com \\ 3Universidade do Estado da Bahia. Salvador, Bahia, Brasil. ORCID: 0000-0003-4853-4151.brenda_machado@hotmail.com \\ ${ }^{4}$ Universidade do Estado da Bahia. Salvador, Bahia, Brasil. ORCID: 0000-0003-4914-7698. carlosandrejpa@gmail.com \\ ${ }^{5}$ Fundação de Hematologia e Hemoterapia da Bahia - HEMOBA. Salvador, Bahia, Brasil. \\ ORCID: 0000-0003-3828-1023. lucialeandro1@hotmail.com \\ ¿Universidade do Estado da Bahia. Salvador, Bahia, Brasil. ORCID: 0000-0003-4220-1313. macedocezar@bol.com.br
}

\begin{abstract}
RESUMO I INTRODUÇÃO: A hemofilia é uma doença crônica de origem genética causada por uma mutação dos genes que codificam os fatores de coagulação sanguíneos. Uma das consequências dessa alteração é o acometimento do sistema musculoesquelético, o que pode influenciar na postura desses indivíduos. OBJETIVO: Estimar a frequência de alterações posturais em portadores de hemofilia, e identificar possiveis fatores associados. MÉTODOS: Estudo descritivo de corte transversal, conduzido com indivíduos com idade $\geq 18$ anos, com diagnóstico de hemofilia, em acompanhamento na Fundação de Hematologia e Hemoterapia da Bahia (HEMOBA). Os participantes foram submetidos à avaliação postural, por meio da análise visual, teste de Adams e aplicação de um formulário semi-estruturado. Empregou-se o Epi Info® (v.3.5.2) para análise dos dados e, para verificar a existência de associações entre as variáveis do estudo foram utilizados os Testes exato de Fisher, Qui-quadrado (bicaudal) e Qui-Quadrado com a correção de Yates. Consideradas como estatisticamente significantes associações com $p<0,05$. RESULTADOS: Participaram deste estudo 29 hemofílicos, com média de idade de $34,9 \pm 12,6$ anos, $25(86,2 \%)$ informaram ter artropatia hemofílica. Dos participantes da pesquisa $13(68,4 \%)$ apresentaram postura escoliótica detectada pelo teste de Adams. A alteração foi mais frequente nos indivíduos com mais de uma articulação afetada pela artropatia hemofílica, com valor estatisticamente significativo para esta variável $(p=0,039)$. CONCLUSÃO: As frequências de alterações posturais em indivíduos com hemofilia são elevadas com associação significativa para os indivíduos com idade superior a 40 anos e com mais de uma articulação afetada pela artropatia hemofílica.
\end{abstract}

\begin{abstract}
INTRODUCTION: A hemophilia is a chronic disease of genetic origin caused by a mutation of the genes which encode blood clotting factors. One of the consequences of this alteration is the involvement of the musculoskeletal system, which may influence the posture of these individuals. OBJECTIVE: To estimate the frequency of postural changes in hemophilia patients, and identify possible factors associated. METHODS: A cross-sectional descriptive study, conducted with individuals aged $\geq 18$ years, with diagnosis of hemophilia, in follow-up at the Hematology and Hemotherapy Foundation of Bahia (HEMOBA).The participants were submitted to postural evaluation,through visual analysis,

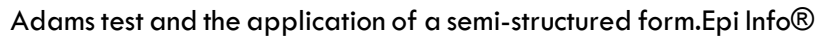
(v.3.5.2) was used for data analysis and to verify the existence of associations between the variables of the study were used the Exact Fisher, Qui-Square and Qui-Square Tests with the Yates correction and considered as statistically significant associations with $p<0.05$. RESULTS: Twenty-nine hemophiliacs participated in this study, with mean age of $34.98 \pm 12,6$ years, $25(86.2 \%)$ reported having hemophilic arthropathy. Of the 13 research participants $(68.4 \%)$ presented scoliotic posture detected by the Adams test.The change was more frequent in individuals with more than one joint affected by hemophilic arthropathy, with a statistically significant value for this variable $(p=0.039)$. CONCLUSION: The frequencies of postural changes in individuals with hemophilia are elevated with a significant association for individuals over 40 years of age and with with more than one joint affected by haemophilic arthropathy.
\end{abstract}

KEYWORDS: Hemophilia. Posture. Hemarthrosis. Bruise. 


\section{Introdução}

A hemofilia é uma doença crônica de origem genética causada por uma mutação dos genes que codificam os fatores de coagulação VIII (hemofilia A) e IX (hemofilia B) ${ }^{1-3}$. Uma das conseqüências dessa alteração é o acometimento do sistema musculoesquelético ${ }^{2,3}$. Existem cerca de $400 \mathrm{mil}$ pessoas acometidas com essa doença no mundo, sendo mais frequente em indivíduos do sexo masculino por seu caráter hereditário ligado ao cromossomo "X". Mulheres carregam o gene da doença, porém raramente manifestam o distúrbio ${ }^{1-3}$. Estima-se no Brasil, cerca de 12 mil portadores, destes, três mil apresentaram a forma grave da doença, levando, em alguns casos, até a invalidez do indivíduo devido às frequentes hemorragias ${ }^{2,4}$.

As hemartroses representam uma das complicações clínicas mais frequentes e preocupantes por ser preditora de limitações funcionais ${ }^{2,3,5}$. $O$ processo degenerativo geralmente é poliarticular que acomete, sobretudo, a articulação dos joelhos e tornozelos ${ }^{2,6,7}$. Já os hematomas ocorrem principalmente no sistema muscular, com maior frequência no músculo iliopsoas ${ }^{2,8,9}$. Deste modo, as alterações posturais, principalmente os desvios anormais da coluna, são uma das complicações que esses indivíduos estão sujeitos ${ }^{7}$, causadas tanto pelas deformidades estruturais, desequilíbrio muscular e/ou contraturas, quanto pelos maus hábitos posturais $^{10,11}$.

A postura é caracterizada como a posição que o corpo adota no espaço, com o objetivo de estabelecer um equilíbrio entre as estruturas de suporte, músculos e ossos ${ }^{12,13}$. Uma boa postura consiste em provocar estresse mínimo sobre articulações e um baixo gasto energético na atividade muscular ${ }^{13}$. $O$ mau alinhamento corporal pode alterar a distribuição de cargas e de pressões nas superfícies articulares, contribuir para a degeneração articular e tensões musculares ${ }^{7,12,13}$. Utiliza-se a avaliação postural como método de diagnóstico das alterações na postura e como parâmetro para monitorização da efetividade de um tratamento e adoção de medidas preventivas $^{14}$.

Diante da gravidade das complicações articulares decorrentes da hemofilia, sua elevada frequência e carência de estudos, no Brasil e particularmente na cidade de Salvador- $\mathrm{Ba}$, sobre a incidência de alterações posturais nesta população, fazse necessária a realização de pesquisa com esta temática. Pelo exposto, o presente estudo tem como objetivo estimar a frequência de alterações posturais em portadores de hemofilia atendidos no Centro de Referência de Hematologia e Hemoterapia da Bahia, e identificar possíveis fatores associados.

\section{Material e métodos}

Trata-se de um estudo de corte transversal descritivo conduzido com pacientes maiores de 18 anos, com diagnóstico de hemofilia, acompanhados na Fundação de Hematologia e Hemoterapia da Bahia (HEMOBA), localizada na cidade de Salvador, Bahia. A coleta foi realizada no período de agosto de 2016 a março de 2017. Foram incluídos todos que estavam em acompanhamento regular no HEMOBA, presentes no período da coleta e que aceitaram participar do estudo. Foram excluídos pacientes com alterações posturais provenientes de outras doenças e aqueles que apresentassem relato de doenças musculoesqueléticas e neurológicas diagnosticadas previamente ou episódio de hemartrose/hematoma há menos de 15 dias da coleta dos dados devido o quadro de dor e edema.

A avaliação postural e coleta dos dados foram realizadas pelos pesquisadores previamente treinados. Foi realizado um estudo piloto, no qual foi aplicado um instrumento semi- estruturado elaborado pelos autores e realizada a avaliação postural por meio da análise visual. $O$ estudo piloto foi realizado com cinco portadores de hemofilia acompanhados pelo Hemoba, no período de uma semana. Com os objetivos de calibrar e aperfeiçoar o instrumento da coleta e uniformizar a avaliação postural. $O$ instrumento semi-estruturado apresentava as variáveis sociodemográficas, clínicas e antropométricas.

No que diz respeito às variáveis sociodemográficas, questionou-se idade, em anos completo, a cor da pele de acordo com o IBGE, colapsadas em branca ou não branca, escolaridade, situação conjugal e atividade laboral ${ }^{15}$. Em relação às variáveis antropométricas investigou-se $\circ$ peso corporal em $\mathrm{kg}$, altura em metros, circunferência abdominal e o 
Índice de Massa Corpórea (IMC). A partir dos valores do IMC foi possível obter a classificação percentil de cada participante, e sua categorização de peso em magreza/eutrófico e sobrepeso/obesidade.

Foi incluindo na avaliação o tipo de hemofilia, se A ou $B$, classificadas de acordo com a deficiência do fator VIII ou IX, respectivamente. O grau de hemofilia foi inserido segundo a sua gravidade correlacionada a apresentação da atividade do fator de coagulação, se abaixo de $1 \%$, grave, entre 1 a $5 \%$, moderada ou entre 6 a $30 \%$, leve. Foram questionados, neste estudo, a presença ou não de inibidor do fator de coagulação e o tipo de reposição de fator, se por demanda ou profilaxia'. Avalio-se também, a presença de artropatia hemofílica e quantas articulações são acometidas: apenas uma ou mais. Questionou-se a presença de articulação alvo, deformidades em flexão ou extensão e a realização de acompanhamento fisioterapêutico. A lateralidade foi definida como destro ou canhoto. Considerouse ativo fisicamente aquele que afirmou praticar atividade física ao menos três vezes na semana.

Para a avaliação postural os pacientes foram orientados a usar roupas apropriadas, como short, no caso dos homens, e short e top para as mulheres. Realizou-se a avaliação postural dos sujeitos da pesquisa em posição ortostática em vista anterior, lateral e posterior, de forma subjetiva, por meio da analise visual ${ }^{14}$. Foi utilizado também, um formulário semi estruturado, no qual, as informações observadas foram assinaladas nas opções padronizadas relativas à observação. Posteriormente foram realizadas fotografias dos participantes, nas mesmas posições da avaliação postural, afim de confirmação dos achados relacionados à avaliação postural, pelo mesmo examinador que realizou a analise visual. A fotografia digital foi realizada com a câmera do celular Samsung ${ }^{\circledR}$ J5, 13 megapixels, resolução de $4128 \times 3096$ pixels com utilização de flash e sem utilização de zoom. O celular foi posicionado a dois metros e meio de distância dos voluntários e a um metro de altura do chão, o celular ficou perpendicular ao indivíduo ${ }^{16}$.

$\mathrm{Na}$ vista anterior, foi avaliada a presença ou ausência de assimetria das cinturas escapular e pélvica, se há nivelamento entre os maléolos mediais e o posicionamento dos tornozelos, se neutros, pronados ou supinados. Foi observado
○ ângulo de tales, se é simétrico, maior à direita ou maior à esquerda. Em uma vista lateral foi avaliada a posição da cabeça, se centralizada, anteriorizada ou estendida; 0 posicionamento dos ombros, se alinhados em antepulsão ou retropulsão; o alinhamento dos joelhos, se alinhados, recurvados ou flexionados; e se existe aumento ou diminuição da cifose torácica e da lordose lombar. Na vista posterior verificou-se a simetria dos joelhos através da interlinha articular, se nivelados, maior a direita ou maior a esquerda.

O Teste de Adams foi selecionado para verificar a presença de gibosidades. Para realização do teste os participantes foram solicitados a ficarem na posição ortostática, descalços, com pés juntos, joelhos estendidos, e inclinarem o tronco para frente tentando tocar os dedos dos pés. $O$ teste de Adams foi considerado positivo quando houve presença de gibosidade ou desvio do eixo da coluna vertebral pela projeção das apófises espinhosas ${ }^{14}$.

O comprimento dos membros inferiores foi verificado de acordo com o protocolo de Magge, utilizando fita métrica da marca Universal Medical Products INC ${ }$, com os voluntários posicionados em decúbito dorsal sobre uma maca, com rotação neutra de quadril e maléolos mediais o mais próximos da linha sagital corporal. Em seguida, o examinador, posicionado ao lado do membro a ser medido, localizava, por meio da palpação, os pontos anatômicos da espinha ilíaca ântero-superior e ponto mais distal e medial do maléolo medial do mesmo lado. Com auxílio de uma fita métrica, mediu-se de um ponto a outro, registrando o resultado em centímetros ${ }^{14}$. O tipo de pé, se neutro, plano ou cavo foi avaliado com - auxilio do podoscópio da marca Cris Medical®: Modelo podoscópio ref.552.915.

O banco de dados foi criado no programa Microsoft Office Excel 2007 e para análise dos dados foi empregado o Epi Info® (versão 3.5.2). Para cálculo das medias, desvio padrão, frequências absoluta e relativa para descrição das variáveis de interesse. A associação entre as variáveis do estudo foi verificada por meio dos Testes exato de Fisher Qui-quadrado (bicaudal)e Qui-Quadrado com a correção de Yates. Considerou-se como estatisticamente significantes associações com $p<0,05$ (IC 95\%).

O projeto de pesquisa foi aprovado pelo Comitê de Ética em Pesquisa da Universidade do Estado 
da Bahia (Registro 055417/2016 CEP-UNEB, em 13/06/2016; CAAE 56930116.9.0000.0057) em conformidade com as normas da Resolução 466/12 do Conselho Nacional de Ética em Pesquisa (CONEP).

\section{Resultados}

Foram 37 indivíduos hemofílicos, acompanhados pela fundação de Hemoterapia e Hematologia da Bahia (HEMOBA). Destes, três $(8,1 \%)$ não atendiam aos critérios de inclusão e cinco $(10,8 \%)$ se recusaram a participar da pesquisa, totalizando 29 indivíduos.

Na Tabela 1 são apresentadas as características sociodemográficas dos hemofílicos. A amostra foi composta exclusivamente pelo sexo masculino $29(100 \%), 22(75,9 \%)$ encontravam-se na faixa etária dos 18 a 40 anos com média de idade $34,9 \pm 12,6$ anos, $22(75,9 \%)$ consideravam-se não brancos. Quanto à escolaridade, $23(79,3 \%)$ tinham o ensino médio/superior completo ou incompleto, $15(51,7 \%)$ trabalhavam e $21(72,5 \%)$ viviam sem companheira.

Tabela 1. Características sociodemográficas e antropométrica de hemofílicos acompanhados pelo HEMOBA. Salvador, Bahia, 2017.

\begin{tabular}{|c|c|c|c|c|}
\hline Variáveis & $n=29$ & $\%$ & Média & DP \\
\hline \multicolumn{5}{|l|}{ Sexo } \\
\hline Masculino & 29 & 100 & - & - \\
\hline Feminino & - & - & & \\
\hline \multicolumn{5}{|l|}{ Idade } \\
\hline $18-40$ & 22 & 75,9 & 34,9 & 12,6 \\
\hline$>40$ & 7 & 24,1 & & \\
\hline \multicolumn{5}{|l|}{ Cor da pele } \\
\hline Branca & 7 & 24,1 & & \\
\hline Não branca & 22 & 75,9 & - & - \\
\hline \multicolumn{5}{|l|}{ Escolaridade } \\
\hline $\begin{array}{l}\text { Analfabeto/ensino } \\
\text { fundamental }\end{array}$ & 6 & 20,7 & & \\
\hline $\begin{array}{l}\text { Ensino médio/superior } \\
\text { completo ou incompleto }\end{array}$ & 23 & 79,3 & - & - \\
\hline \multicolumn{5}{|l|}{ Atividade laboral } \\
\hline Trabalha & 15 & 51,7 & - & - \\
\hline Não trabalha & 6 & 20,7 & & \\
\hline Aposentado /Beneficiário & 8 & 27,6 & & \\
\hline \multicolumn{5}{|l|}{ Situação conjugal } \\
\hline Vive com parceira (o) & 8 & 27,5 & & \\
\hline Vive sem parceira (o) & 21 & 72,5 & - & - \\
\hline
\end{tabular}

Fonte: Dados da pesquisa. DP=Desvio Padrão.

Quanto a classificação do tipo de hemofilia, $22(75,9 \%)$ eram do tipo A, Com relação a gravidade da doença, $21(72,4 \%)$ foram classificados com a forma grave da doença como graves, $21(72,4 \%)$ relataram não ter a presença do inibidor do fator da coagulação e $15(51,7 \%)$ faziam reposição profilática do fator. A artropatia hemofilia em uma ou mais articulações, foi relatada por $25(86,2 \%)$ indivíduos, $15(51,7 \%)$ realizavam tratamento fisioterapêutico e $23(79,3 \%)$ não praticavam atividade física regular. Quanto ao índice de massa corpórea (IMC), 15 (51,7\%) eram magros ou eutróficos. (Tabela 2). 
Tabela 2. Características clínicas de hemofílicos pelo HEMOBA. Salvador, Bahia, 2017.

\begin{tabular}{|c|c|c|}
\hline Variáveis & $n=29$ & $\%$ \\
\hline \multicolumn{3}{|l|}{ Tipo de hemofilia } \\
\hline A & 22 & 75,9 \\
\hline B & 7 & 24,2 \\
\hline \multicolumn{3}{|l|}{ Grau } \\
\hline Leve & 2 & 6,9 \\
\hline Moderado & 6 & 20,7 \\
\hline Grave & 21 & 72,4 \\
\hline \multicolumn{3}{|l|}{ Presença de inibidor } \\
\hline Sim & 8 & 27,6 \\
\hline Não & 21 & 72,4 \\
\hline \multicolumn{3}{|l|}{ Tipo de reposição de fator } \\
\hline Demanda & 14 & 48,3 \\
\hline Profilaxia & 15 & 51,7 \\
\hline \multicolumn{3}{|l|}{ Presença de artropatia } \\
\hline $\operatorname{Sim}$ & 25 & 86,2 \\
\hline Não & 4 & 13,8 \\
\hline \multicolumn{3}{|l|}{$\begin{array}{l}\text { Faz acompanhamento de } \\
\text { fisioterapia }\end{array}$} \\
\hline Sim & 15 & 51,7 \\
\hline Não & 14 & 48,3 \\
\hline \multicolumn{3}{|l|}{ Pratica de atividade física } \\
\hline Sim & 6 & 20,7 \\
\hline Não & 23 & 79,3 \\
\hline \multicolumn{3}{|l|}{ IMC } \\
\hline Magros/estróficos & 15 & 51,7 \\
\hline Sobrepeso/obesos & 14 & 48,3 \\
\hline
\end{tabular}

Fonte: Dados da pesquisa. IMC= Índice de massa corporal.

Na Tabela 3 estão descritas as frequências de alterações posturais em hemofílicos. A deformidade em flexão de um ou ambos os joelhos corresponderam a 20 (69\%), 20 (69\%) apresentaram diminuição da alteração na altura do arco plantar, $20(69 \%)$ tinham uma diferença no comprimento dos membros inferiores maior que um centímetro e 11 (37,9\%) apresentaram alteração de pronação do tornozelo. Aumento da lordose lombar foi verificado em 20 (69\%) indivíduos, e o aumento da cifose torácica em 15 (51,7\%). O teste de Adams foi negativo em $15(51,7 \%)$ hemofílicos e 21 (72,4\%) apresentaram assimetria do ângulo de Tales. 


\begin{tabular}{|c|c|c|}
\hline Variáveis & $n=29$ & $\%$ \\
\hline \multicolumn{3}{|l|}{ Joelhos flexionados } \\
\hline Não & 9 & 31 \\
\hline Sim & 20 & 69 \\
\hline \multicolumn{3}{|l|}{ Tipo de pé } \\
\hline Alinhados & 3 & 10,3 \\
\hline Planos & 20 & 69 \\
\hline Cavos & 6 & 20,7 \\
\hline \multicolumn{3}{|c|}{ Comprimento de MMII } \\
\hline$\leq 1 \mathrm{~cm}$ & 9 & 31 \\
\hline$>1 \mathrm{~cm}$ & 20 & 69 \\
\hline \multicolumn{3}{|l|}{ Tornozelo } \\
\hline Alinhados & 10 & 34,5 \\
\hline Pronados & 11 & 37,9 \\
\hline Supinados & 8 & 27,6 \\
\hline \multicolumn{3}{|l|}{ Lordose lombar } \\
\hline Fisiológica & 9 & 31 \\
\hline Aumentada & 20 & 69 \\
\hline Diminuída & 0 & 0 \\
\hline \multicolumn{3}{|l|}{ Cifose torácica } \\
\hline Fisiológica & 14 & 48,3 \\
\hline Aumentada & 15 & 51,7 \\
\hline Diminuída & 0 & 0 \\
\hline \multicolumn{3}{|l|}{ Teste de Adams } \\
\hline Positivo & 14 & 48,3 \\
\hline Negativo & 15 & 51,7 \\
\hline \multicolumn{3}{|l|}{ Ângulo de Tales } \\
\hline Simétrico & 8 & 27,6 \\
\hline Assimétrico & 21 & 74,4 \\
\hline
\end{tabular}

Fonte: Dados da pesquisa.

Na Tabela 4 encontram-se dispostos os resultados da associação entre o teste de Adams e as variáveis sociodemográficas, clínicas e posturais dos hemofílicos. A presença de postura escoliótica, detectada pelo teste de Adams foi mais frequente na faixa etária $>40$ anos, com valor estatisticamente significante $(p=0,031) r$ ?. Outra variável que também revelaram associação estatisticamente significativa com o teste de Adams foi à quantidade de articulações com artropatia hemofilia, valor estatisticamente significante $(p=0,039)$. Não houve outra relação estatisticamente significante entre as variáveis. 
Tabela 4. Associação entre as variáveis sociodemográficas, clínicas e posturais com o teste de Adams em hemofílicos acompanhados pelo HEMOBA. Salvador, Bahia, 2017.

\begin{tabular}{llccccc}
\hline Variáveis & \multicolumn{2}{c}{$\begin{array}{c}\text { Teste de Adams } \\
\text { Positivo }\end{array}$} & $\begin{array}{c}\text { Teste de Adams } \\
\text { Negativo }\end{array}$ & \multirow{2}{*}{$\begin{array}{c}\text { Valor } \\
\mathbf{P}\end{array}$} \\
\cline { 3 - 5 } & \multicolumn{2}{c}{$\mathbf{n}$} & $\%$ & $\mathbf{n}$ & $\%$ & \\
\hline Idade & & & & & & \\
& $18-40$ & 8 & 36,4 & 14 & 63,6 & $0,031^{*}$ \\
$>40$ & 6 & 85,7 & 1 & 14,3 &
\end{tabular}

IMC

$\begin{array}{lccccc}\text { Magro/ eutrófico } & 6 & 40 & 9 & 60 & 0,364 \\ \begin{array}{l}\text { Sobrepeso/ } \\ \text { Obesidade }\end{array} & 8 & 57,1 & 6 & 42,9 & \end{array}$

Lateralidade

$\begin{array}{lccccc}\text { Destro } & 12 & 46,2 & 14 & 53,8 & 0,473 \\ \text { Canhoto } & 2 & 66,7 & 1 & 33,3 & \end{array}$

\section{Atividade física}

$\begin{array}{lccccc}\text { Sim } & 2 & 33,3 & 4 & 66,7 & 0,360 \\ \text { Não } & 12 & 52,2 & 11 & 47,8 & \end{array}$

Ângulo de tales

$\begin{array}{lccccc}\text { Simétrico } & 2 & 25 & 6 & 75 & 0,128 \\ \text { Assimétrico } & 12 & 57,1 & 9 & 42,9 & \end{array}$

Joelhos flexionados

$\begin{array}{lccccc}\text { Não } & 2 & 22,2 & 7 & 77,8 & 0,067 \\ \operatorname{Sim} & 12 & 60,0 & 8 & 40 & \end{array}$

Comprimento de MMII

$\begin{array}{lccccc}\leq 1 \mathrm{~cm} & 2 & 25 & 7 & 75 & 0,104 \\ >1 \mathrm{~cm} & 12 & 57,1 & 8 & 42,9 & \end{array}$

$\mathbf{N}^{\circ}$ de articulações pela artropatia hemofílica

\begin{tabular}{lccccc} 
Apenas 1 & 1 & 16,7 & 5 & 83,3 & $0,039^{*}$ \\
+1 & 13 & 68,4 & 6 & 31,6 & \\
\hline
\end{tabular}

Fonte: Dados da pesquisa. Teste exato de Fisher. ${ }^{*}$ Valor de $p<0,05$. 
Tabela 5. Freqüência de alterações posturais em indivíduos com hemofilia em acompanhamento (HEMOBA), de acordo com o grau hemofilia. Salvador-Bahia, 2017.

\begin{tabular}{|c|c|c|c|c|c|}
\hline \multirow[t]{2}{*}{ Variáveis } & \multicolumn{2}{|c|}{ Hemofilia leve } & \multicolumn{2}{|c|}{$\begin{array}{c}\text { Hemofilia } \\
\text { grave/moderada }\end{array}$} & \multirow[t]{2}{*}{ Valor de $p$} \\
\hline & $\mathbf{n}$ & $\%$ & $\mathbf{n}$ & $\%$ & \\
\hline \multicolumn{6}{|l|}{ Ângulos de tales } \\
\hline Simétrico & 1 & 12,5 & 7 & 87,5 & $0,482^{* *}$ \\
\hline Assimétrico & 1 & 4,8 & 20 & 95,2 & \\
\hline \multicolumn{6}{|l|}{ Joelhos flexionados } \\
\hline Não & 0 & 0 & 9 & 100 & $0,946^{*}$ \\
\hline Sim & 2 & 10 & 18 & 90 & \\
\hline \multicolumn{6}{|l|}{ Tornozelo } \\
\hline Alinhados & 1 & 10 & 9 & 90 & $0,578^{* *}$ \\
\hline Desalinhados & 1 & 5,3 & 18 & 94,7 & \\
\hline \multicolumn{6}{|l|}{ Comprimento de MMII } \\
\hline$\leq 1 \mathrm{~cm}$ & 1 & 11,1 & 8 & 88,9 & $0,532^{* *}$ \\
\hline$>1 \mathrm{~cm}$ & 1 & 5 & 19 & 95 & \\
\hline \multicolumn{6}{|l|}{ Lordose lombar } \\
\hline Fisiológica & 0 & 0 & 9 & 100 & $0,084^{*}$ \\
\hline Aumentada & 2 & 10 & 18 & 90 & \\
\hline \multicolumn{6}{|l|}{ Cifose torácica } \\
\hline Fisiológica & 1 & 7,1 & 13 & 92,9 & $0,701^{* *}$ \\
\hline Aumentada & 1 & 6,7 & 14 & 93,3 & \\
\hline \multicolumn{6}{|l|}{ Teste de Adams } \\
\hline Positivo & 0 & 0 & 14 & 100 & $0,494^{7}$ \\
\hline Negativo & 2 & 13,3 & 13 & 86,7 & \\
\hline
\end{tabular}

Fonte: Dados da pesquisa. **Teste exato de Fisher. ¥Teste Qui-Quadrado com a correção de Yates.

Na tabela 5 são apresentas as freqüências das alterações posturais em hemofílicos em acompanhamento no HEMOBA de acordo com o grau de hemofilia. Verificou-se que não houve diferença estatisticamente significante quando analisado o ângulo de tales $(p=0,482)$, joelhos flexionados $(p=0,946)$, alinhamento do Tornozelo $(p=0,578)$, diferença do comprimento dos membros inferiores $(p=0,532)$, bem como as variáveis, , alteração da lordose lombar $(p=0,084)$, alteração da Cifose torácica $(p=0,701)$ e Teste de Adams $(p=0,494)$. Também não houve diferença estatística nas variáveis posturais, em relação ao tipo de reposição de fator (Tabela 6). 


\begin{tabular}{|c|c|c|c|c|c|}
\hline \multirow[t]{2}{*}{ Variáveis } & \multicolumn{2}{|c|}{ Demanda } & \multicolumn{2}{|c|}{ Profilaxia } & \multirow{2}{*}{ Valor de $\mathrm{F}$} \\
\hline & $\mathbf{n}$ & $\%$ & $\mathbf{n}$ & $\%$ & \\
\hline \multicolumn{6}{|l|}{ Ângulos de tales } \\
\hline Simétrico & 4 & 50 & 4 & 50 & $0,457^{* *}$ \\
\hline Assimétrico & 10 & 47,6 & 11 & 52,4 & \\
\hline \multicolumn{6}{|l|}{ Joelhos flexionados } \\
\hline Não & 6 & 66,7 & 3 & 33,3 & $0,495^{* *}$ \\
\hline Sim & 8 & 40 & 12 & 60 & \\
\hline \multicolumn{6}{|l|}{ Tornozelo } \\
\hline Alinhados & 5 & 50 & 5 & 50 & $0,894^{\dagger}$ \\
\hline Desalinhados & 9 & 47,4 & 10 & 52,6 & \\
\hline \multicolumn{6}{|l|}{ Comprimento de MMII } \\
\hline$\leq 1 \mathrm{~cm}$ & 4 & 44,4 & 5 & 55,6 & $0,550^{\dagger}$ \\
\hline$>1 \mathrm{~cm}$ & 10 & 50 & 10 & 50 & \\
\hline \multicolumn{6}{|l|}{ Lordose lombar } \\
\hline Fisiológica & 3 & 33,3 & 6 & 66,7 & $0,249^{* *}$ \\
\hline Aumentada & 11 & 55 & 9 & 45 & \\
\hline \multicolumn{6}{|l|}{ Cifose torácica } \\
\hline Fisiológica & 8 & 57,1 & 6 & 42,9 & $0,364^{\dagger}$ \\
\hline Aumentada & 6 & 40 & 9 & 60 & \\
\hline \multicolumn{6}{|l|}{ Teste de Adams } \\
\hline Positivo & 6 & 42,9 & 8 & 57,1 & 0,579 \\
\hline Negativo & 8 & 53,3 & 7 & 46,7 & \\
\hline
\end{tabular}

Fonte: Dados da pesquisa. **Teste exato de Fisher. †Teste Qui-Quadrado.

\section{Discussão}

Nesta pesquisa a frequência de alterações posturais em indivíduos com hemofilia mostrou-se elevada. As principais alterações encontradas foram deformidade em flexão dos joelhos, diminuição da altura do arco plantar, aumento da lordose lombar, aumento do ângulo de Tales e postura escoliótica. Estes achados corroboram com os reportados por Boccalandro et al. ${ }^{17}$, em estudo realizado em Milão, Itália, envolvendo crianças com e sem hemofilia, no qual, a frequência de alterações posturais foi mais elevada naquelas portadoras da doença. Todavia, os autores não descrevem quais as alterações posturais foram encontradas.

Neste estudo, o sexo da amostra foi exclusivamente masculino, este achado se assemelha com os resultados apresentados por Garbin et al. ${ }^{18} \mathrm{em}$ um estudo realizado no hospital-escola no Interior do Estado de São Paulo. Uma possível explicação para estes achados pode ser devido à hemofilia ser rara no sexo feminino, já que para isso ambos os cromossomos X deveriam ser afetados'. Quanto à idade, observou-se uma maior frequência nos adultos jovens, resultados similares foram encontrados por Garbin et al. ${ }^{18}$ no estudo supracitado. Essa baixa frequência de indivíduos mais velhos pode ser consequência da baixa expectativa de vida desses indivíduos antigamente, pois o tratamento oferecido era limitado.

Em relação à atividade laboral, foi observado que a maioria dos indivíduos trabalhava, porém, vale salientar que por se tratar de uma população composta predominantemente por adultos jovens, uma parcela significante desta população encontrava-se na situação de beneficiários ou aposentados pela doença. Resultados similares foram reportados por Caio et al. ${ }^{19} \mathrm{em}$ um estudo realizado com hemofílicos, acompanhados regularmente no Hemocentro da Universidade Estadual de Campinas - UNICAMP, Campinas, São Paulo. Os autores compararam indivíduos hemofílicos com seus irmãos sem a doença, respeitando a faixa etária. Foi possível verificar que os maiores empecilhos dos portadores de hemofilia 
são de natureza sócio-econômica. Além das reduzidas chances de acessos ao emprego regular e maior taxa de aposentadoria, provavelmente por invalidez.

A prática de exercícios físicos pode contribuir para melhor qualidade de vida e saúde, aumentando a força muscular, função articular, o equilíbrio e a flexibilidade. Apesar disso, a maioria dos participantes deste estudo não praticava atividades físicas regulares. Este achado corroborou com os resultados do estudo de Nunes et al. ${ }^{20}$, no qual, os autores categorizaram a qualidade de vida de hemofílicos que estavam sendo acompanhados em um hemocentro regional de São Paulo. Estes resultados divergiram dos encontrados por Von Mackensene et al. ${ }^{21}$ em estudo realizado em quatro centros de hemofilia em todo o Reino Unido que demonstraram uma maior frequência de indivíduos que realizavam atividade física, incluindo esportes de contato, e estes apresentavam uma menor taxa de alterações articulares. Uma possível explicação para essa divergência pode ser a cultura dos países desenvolvidos de promover a profilaxia primaria na infância, evitando assim maiores danos articulares.

Este estudo também evidenciou uma alta frequência de deformidades em flexão de joelhos. Estes achados podem estar associados à elevada frequência de artropatias hemofílicas nos indivíduos dessa pesquisa. Galante et al. ${ }^{10} \mathrm{em}$ estudo com crianças hemofílicas apresentando ou não sinovite, na faixa etária de 3-7 anos de idade, reforçaram esta associação entre comprometimento articular e alteração postural. Eles demonstraram que crianças com sinovite apresentam uma diminuição do ângulo do joelho (semiflexão) em relação a crianças sem sinovite. Além disso, afirmaram que quanto mais global e precoce for à alteração articular no hemofílico, maior a chance de haver prejuízos no alinhamento articular. $\bigcirc$ que indica a importância da avaliação postural precoce, pois quando os problemas ortopédicos não são corrigidos na infância podem levar às limitações na vida adulta.

Santos et al. ${ }^{11}$ também evidenciaram uma elevada frequência de deformidade em flexão dos joelhos, em estudo realizado com hemofílicos acompanhados no Centro de Hemoterapia e Hematologia do Espírito Santo. Neste estudo, foi constatado que as articulações mais afetadas, por deformidades foram os joelhos. Dados semelhantes foram demonstrados no estudo de Bastos et al. ${ }^{22}$ realizado no Vale do Paraíba Paulista, por meio de uma pesquisa do tipo retrospectiva, documental descritiva. Foi realizado levantamento de dados nos prontuários, de indivíduos com hemofilia, do Ambulatório de Hematologia. Os autores reportaram alta incidência de hemartrose nessas articulações. $O$ que pode ser sugestivo de contratura em flexão dos joelhos, visto que, as frequências de hemartroses favoreceram o quadro de artropatia hemofílica, sendo a contratura em flexão dos joelhos uma das suas complicações.

O aumento da lordose lombar foi verificado na maioria dos indivíduos participantes deste estudo. Apesar de não ter encontrado estudos que descrevessem especificamente esse comprometimento na coluna lombar em hemofílicos, Galatro et al. ${ }^{9}$ afirmaram que os hematomas favorecem o encurtamento e/ ou fibrose muscular. Quando ocorrem no músculo iliopsoas associado às frequentes hemartroses nos joelhos e ao quadro álgico, geralmente imprimem ao indivíduo a adoção de postura antálgica de descanso, com os joelhos fletidos, anteversão pélvica e consequente hiperlordose. Kim et al. ${ }^{23}$ investigaram a relação entre a força muscular do tronco e a lordose lombar em 31 indivíduos sem hemofilia, com média de idade de 35 anos. Os autores observaram que o desequilíbrio na força muscular do tronco pode influenciar significativamente a curva lordótica da coluna lombar. Estes achados podem justificar a elevada frequência de alterações na coluna lombar, visto que, para a manutenção da postura ortostática, há necessidade de músculos fortes, flexíveis e facilmente adaptáveis às alterações do ambiente.

A presença da postura escoliótica, identificada por meio do teste de Adams positivo, associou-se significativamente com a quantidade de articulações acometidas pela artropatia hemofílica. Resultado que condiz com a literatura ${ }^{11}$. Hoepers ${ }^{24}$ reitera com seu estudo conduzido com hemofílicos residentes no Estado de Santa Catarina, no qual, foi observado que à artropatia hemofílica, acomete principalmente as articulações dos joelhos. A artropatia hemofílica gera um quadro de contraturas fixas em flexão dessas articulações, favorecendo uma resposta compensatória de flexão de quadril e uma posição em plante flexão do tornozelo, podendo levar a uma postura escoliótica ${ }^{11}$. Não foram encontrados outros estudos que realizaram essa correlação. 
Observa-se uma presença elevada de postura escoliótica, detectada pelo Teste de Adams, nos indivíduos hemofílicos maiores de 40 anos de idade. Esse achado pode ser justificado, pela existência de uma maior dificuldade no acesso ao tratamento dessa doença antigamente, que favorece a uma maior ocorrência de alterações no sistema musculoesquelético. Não foram encontrados outros estudos que realizaram essa correlação com indivíduos com hemofilia.

Não foi possível estabelecer uma relação estatística significante entre as variáveis de alterações posturais e o grau de hemofilia. Porém, foi possivel observar que nos hemofílicos com hemofilia grave/ moderada as alterações posturais eram mais frequentes, principalmente as alterações relacionadas à lordose lombar. Esses achados podem estar associados à maior predisposição de danos articulares e musculares que esses indivíduos estão sujeitos ${ }^{5}$. Também não foram encontrados outros estudos quantitativos realizado com a mesma população, do presente estudo, que observassem esta relação.

Apesar da reposição de fator, pela profilaxia primária ou secundária, ser uma forma preventiva de complicações articulares não houve associações estatísticas entre o tipo de reposição de fator, se por demanda ou profilaxia, com as frequências de alterações posturais. Esse resultado pode ser explicado devido à implementação tardia, no Brasil, da profilaxia primária, pelo Ministério da Saúde (MS) em dezembro de 2011 e a profilaxia secundária em $2012^{25}$. É importante ressaltar também, que o tempo em anos que os hemofílicos, desta pesquisa, relataram fazer a profilaxia não foi suficiente para predizer um padrão postural diferenciado ou para reverter alterações posturais que se instalaram com o passar dos anos, visto que, este não é o objetivo da profilaxia. Não foram encontrados estudos que contribuíssem com dados semelhantes.

emprego do desenho transversal impossibilita inferências causais, muito embora tal metodologia tenha relevância para caracterizar a população. O baixo custo e fácil acesso para coleta de dados foram vantagens desse estudo, porém, a não realização de um cálculo amostral o número amostral reduzido pode ser apontado como uma fragilidade uma vez que não possibilita uma extrapolação de resultados para indivíduos com hemofilia. Outra questão a ser considerada é o fato das avaliações posturais terem sido realizadas por diferentes avaliadores e por meio da analise visual, fato que pode ter gerado um viés de aferição. Para minimizar este viés, foi realizado treinamento prévio com a equipe de avaliadores, além da realização de um estudo-piloto.

\section{Conclusão}

Os resultados obtidos neste estudo demonstraram elevada frequência de alterações posturais, principalmente membros inferiores e nas curvaturas da coluna, com associação significativa para os indivíduos com idade superior a 40 anos e com mais de uma articulação afetada pela artropatia hemofílica. Estes achados podem contribuir para justificar a necessidade de implantação de programas de prevenção e rastreamento precoce pelos profissionais e gestores de saúde que trabalham diretamente com essa população.

Estas ações podem ajudar a diminuir a incidência destes agravos, além de promover a detecção precoce das alterações, que é fundamental para prevenção de complicações futuras que venham comprometer a qualidade de vida desses indivíduos e sua funcionalidade. No entanto, a realização de novas pesquisas a respeito das alterações posturais nessa população, com um grupo controle com indivíduos hígidos e a associação de instrumentos de avaliação postural, deve ser encorajada. Sendo plausivel reforçar a necessidade de implantação destes programas quanto para investigar outras variáveis associadas.

\section{Contribuições dos autores}

Santos RS participou da concepção, delineamento, busca e análise estatística dos dados da pesquisa, interpretação dos resultados, redação do artigo científico e encaminhamento do artigo científico. Figueirôa GR participou do delineamento, busca e análise estatística dos dados da pesquisa, interpretação dos resultados e redação do artigo. Machado BA participou da busca e análise estatística dos dados da pesquisa, interpretação dos resultados e redação do artigo. Mamede CA participou do delineamento, busca e análise estatística dos dados da pesquisa, interpretação dos resultados. 
Gois LML participou do delineamento, busca e análise estatística dos dados da pesquisa, interpretação dos resultados; e Júnior Silva MCM participou da busca e análise estatística dos dados da pesquisa e interpretação dos resultados.

\section{Conflitos de interesses}

Nenhum conflito financeiro, legal ou político envolvendo terceiros (governo, empresas e fundações privadas, etc.) foi declarado para nenhum aspecto do trabalho submetido (incluindo mas não limitandose a subvenções e financiamentos, conselho consultivo, desenho de estudo, preparação de manuscrito, análise estatística, etc).

\section{Referências}

1. Arruda VR, High KA. Distúrbios da coagulação. In: Longo DL. Hematologia e Oncologia de Harrison. 2.ed. Porto Alegre: AMGH; 2015. p.193-197.

2. Brasil. Ministério da Saúde. Secretaria de Atenção à Saúde. Departamento de Atenção Especializada. Manual de Reabilitação na hemofilia. Brasília: Ministério da Saúde; 2011 .

3. Paula EV, Villaça PR. Abordagem clínica da hemofilia. In: Pacheco LRL, Wolff ALP. Ortopedia e fisioterapia em hemofilia. Barueri: Manole; 2013. p.19-37.

4. Ministério da Saúde. Secretaria de Atenção à Saúde. Departamento de Atenção Especializada e Temática. Perfil das coagulopatias hereditárias no Brasil: 2014. Brasília: Ministério da Saúde; 2015.

5. Rodríguez-Merchán EC, Rodríguez HC. Fisiopatologia dos problemas musculoesqueléticos da hemofilia. In: Pacheco LRL, Wolff ALP. Ortopedia e fisioterapia em hemofilia. Barueri: Manole; 2013. p.59-70.

6. Lagunas RB. Hemartrose aguda. In: Pacheco LRL, Wolff ALP. Ortopedia e fisioterapia em hemofilia. Barueri: Manole; 2013.

7. Cunha LAM, Gois EJA. Deformidades angulares na hemofilia. In: Pacheco LRL, Wolff ALP. Ortopedia e fisioterapia em hemofilia. Barueri: Manole; 2013. p.291-303.

8. Beeton K, Rodriguez-Merchan EC, Alltree J, Cornwall J. Rehabilitation of Muscle Dysfunction in Hemophilia. Treatment of Hemophilia. 2012;(24):1-12.

9. Galatro G, Daffunchio C, Moretti NG. Hematomas musculares: diagnóstico e tratamento. In: Pacheco LR, Wolff ALP. Ortopedia e fisioterapia em hemofilia. Barueri: Manole; 2013. p. 147-173.

10. Galante GA, Azevedo CSA, Mello M, Tanaka C, D'Amico EA. Avaliação do alinhamento postural e do desempenho em atividades funcionais de crianças hemofílicas em idade inferior a sete anos, com e sem sinovite crônica: correlação com a incidência de hemartroses. Rev bras fisioter. 2006;10(2):171-176.

11. dos Santos EG, Pontes LL, Santana AG, Neto ETS.

Deformidades e incapacidades dos hemofílicos do centro de hematologia e hemoterapia do Espírito Santo, Brasil. Rev Ter Ocup Univ São Paulo. 2006;18(2):86-94. doi: 10.11606/ issn.2238-6149.v18i2p86-94

12. Shumway-Cook A, Woollacott MH. Controle motor teoria e aplicações práticas. 2.ed. Barueri: Manole; 2003.

13. Landim E. Coluna toracolombar: deformidades não paralíticas. In: Sizínio H. Ortopedia e traumatologia: princípios e práticas. 4.ed. Porto Alegre: Artmed; 2009. p. 97-109.

14. Magee DJ. Avaliação musculoesquelética. 5.ed. Barueri: Manole; 2010.

15. Instituto Brasileiro de Geografia e Estatística. Relatório populacional dos municípios brasileiros[Internet]. 2008 [acesso em 2017 mai]. Disponível em: www.ibge.gov.br

16. lunes DH, Castro FA, Salgado HS, Moura IC, de Oliveira AS, Bevilaqua-Grossi D. Confiabilidade inter e intraexaminadores e repetibilidade da avaliação postural pela fotogrametria. Rev Bras Fisioter. 2005;9(3):327-34.

17. Boccalandro E, Pasta G, Mannucci PM, Santogostino E, Peyvandi F, Sueser A et al. Integrated postural analysis in children with haemophilia. Haemophilia. 2014;20(2):263267. doi: 10.1111 /hae. 12369

18. Garbin LM, de Carvalho EC, Canini SRMS, Dantas RAS. Avaliação da qualidade de vida relacionada à saúde em pacientes portadores de hemofilia. Ciência, Cuidado e Saúde. 2007;6(2):197-205. doi: 10.4025/cienccuidsaude. v6i2.4153

19. Caio MV, Silva RBP, Magna LA, Ramalho AS. Genética comunitária e hemofilia em uma população brasileira. Cad Saúde Pública. 2001;17(3):595-605. doi: 10.1590/50102$\underline{311 \times 2001000300014}$

20. Nunes AA, Rodrigues BSC, Soares EM, Soares $S$, Miranzi SSC. Qualidade de vida de pacientes hemofílicos acompanhados em ambulatório de hematologia. Rev Bras Hematol Hemoter. 2009;31(6):437-43. doi: 10.1590/ $\underline{\mathrm{S} 1516-84842009005000085}$

21. Von Mackensen S, Harrington C, Tuddenham E, Littley A, Will $A$, Fareh $M$ et al. The impact of sport on health status, psychological well-being and physical performance of adults with haemophilia. Haemophilia. 2016;22(4):521-530. doi: $10.1111 /$ hae.12912

22. Bastos NA, Cavaglieri AG. Caracterização dos portadores de hemofilia do Vale no Paraíba Paulista-SP. REENVAP, Lorena. 201 2;3(2):55-64. 
23. Kim HJ, Chung S, Kim S, Shin H, Lee J, Kim S et al. Influences of trunk muscles on

lumbar lordosis and sacral angle. Eur Spine J.

2006; 15(4):409-14. doi: 10.1007/s00586-005-0976-5

24. Hoepers, ATC. Hemofilia no estado de Santa Catarina: estudo clínico. Rev Bras Hematol Hemoter. 2008;30(2):172. doi: $10.1590 /$ S1516-84842008000200020

25. Ministério da Saúde. Portal saúde [Internet]. [atualizado em 2015 abril 17; acessado em 2017 abril 29]. Disponível em: http://www.saude.gov.br 